\title{
Sleep duration and cardiovascular risk: results of the large-scale epidemiology study ESSE-RF
}

\author{
Mikhail Bochkarev', Lyudmila Korostovtseva', Elena Medvedeva', Oxana Rotar', Yurii Sviryaev', \\ Yulia Zhernakova ${ }^{3}$, Svetlana Shalnova ${ }^{4}$, Alexandra Konradii, ${ }^{1}$, Irina Chazova, ${ }^{3,6}$, Sergey Boytsov ${ }^{4,7}$, \\ Eugene Shlyakhto ${ }^{1}$
}

\author{
${ }^{1}$ Almazov National Medical Research Centre, St. Petersburg, Russian Federation \\ ${ }^{2}$ Sechenov Institute of Evolutionary Physiology and Biochemistry of RAS, St. Petersburg, Russian Federation \\ ${ }^{3}$ Russian Cardiology Research and Production Complex, Moscow, Russian Federation \\ ${ }^{4}$ National Research Centre for Preventive Medicine, Pirogov University, Moscow, Russian Federation \\ ${ }^{5}$ ITMO University, St. Petersburg, Russian Federation \\ ${ }^{6}$ M. Sechenov First Moscow State Medical University (Sechenov University), Moscow, Russian Federation \\ ${ }^{7}$ National Medical Research Center of Cardiology, Moscow, Russian Federation
}

\begin{abstract}
Background. The recent data suggest that sleep disorders are associated with cardiovascular diseases. We assessed the relation between self-reported sleep duration and cardiovascular and metabolic disorders in the large-scale epidemiological study.

Material and methods. The ESSE-RF is a population-based cross-sectional study involving 22,258 participants aged 25-64 years from 13 regions of the Russian Federation. In 2012-2014, all subjects underwent a structured interview including questions about average daily sleep duration, lifestyle, complaints and diseases. The current analyses considered the associations with the following disorders: obesity, hypertension, coronary artery disease, myocardial infarction, stroke (cerebral thrombosis or hemorrhage) and diabetes mellitus.

Results. Altogether 20,359 respondents were included in the final analysis. The mean self-reported sleep duration was $7.0 \mathrm{~h}$ per night: $23.3 \%$ participants reported sleeping less than $6 \mathrm{~h}$ while $4.5 \%$ subjects slept more than $9 \mathrm{~h}$. We found both short and long sleep duration to be associated with self-reported cardiovascular diseases. The association was independent of age, sex, body mass index, blood pressure, lipids and glucose levels. The multivariable odds were higher for obesity in short-sleepers compared to those sleeping 7-8 h. In the meanwhile, the association was $\mathrm{U}$-shaped for coronary artery disease. A J-shaped relation was found for myocardial infarction. No relation was found for hypertension, diabetes mellitus or stroke.

Conclusions. Differences in sleep duration may have health consequences given associations between short and long sleep duration and cardiometabolic outcomes.

Key words: epidemiology; obesity; sleep; risk factors; cardiovascular diseases
\end{abstract}

Arterial Hypertens. 2019, vol. 23, no. 4, pages: 248-255

DOI: 10.5603/AH.a2019.0017

Address for correspondence: Lyudmila Korostovtseva, MD, PhD

Almazov National Medical Research Centre, St. Petersburg, Russian Federation; e-mail: lyudmila_korosto@mail.ru

V M Copyright $\odot 2019$ Via Medica, ISSN 2449-6170 


\section{Introduction}

Increasing evidence indicates that sufficient sleep duration and sleep quality are important for the primary and secondary cardiovascular prevention $[1,2]$. The leading sleep medical associations recommend 7-9 hours of daily sleep for adults [3-5]. The association between the cardiovascular event risk and sleep duration is characterized by the U-shape curve which implies different mechanisms affecting prognosis in the long- and short-sleepers [6]. Moreover, the majority of cross-sectional and observational studies show an increased rate of obesity, cardiovascular diseases, stroke and mortality in subjects with poor sleep quality, as well as in short- and long-sleepers [7]. A number of experimental studies showed that short sleep is potentially associated with the adverse endocrine, immune, and metabolic effects. However, the underlying mechanisms remain unclear. Nevertheless, the following mechanisms are discussed: sympathetic activation, disruption of energy balance, proinflammatory state, impaired insulin sensitivity, enhancement of lipid metabolism abnormalities, etc. [8-10]. Experimental studies demonstrated blood pressure elevation after one night of partial sleep deprivation [11] and its decline after sleep prolongation [12]. All these factors are known to be involved in the cardiovascular continuum promoting atherosclerosis progression and increasing risk of cardiovascular events [4]. Some authors indicate that different sleep duration and sleep disturbances might be associated with different outcomes. Thus, CARDIA study showed that long sleep duration is mostly associated with increased risk of dyslipidemia [13]. Cross-sectional and prospective studies demonstrated that the prevalence of stroke and myocardial infarction (MI) is higher in both short and long sleepers, but the sleep debt mostly increases risk of MI [7, 14].

Complaints related to short sleep and poor sleep quality are more often reported by elderly people. Some studies showed that these changes are more frequent and start earlier in women than in men [15]. Moreover, based on the Whitehall II Study, only in women short duration of sleep is associated with higher risks of hypertension. The gender-related differences are mostly explained by the effects of sex hormones, and in particular major hormonal shifts more pronounced in women than in men. At the same time, psychosocial and behavioral factors can also have an impact on sleep characteristics in men and women [16].

The multicenter study "Epidemiology of cardiovascular disease in different regions of the Russian Federation - ESSE-RF" is the first epidemiological study to evaluate the sleep duration among Russians [17]. The main objective of this epidemiological study was to study the prevalence of risk factors and cardiovascular diseases in 13 regions of the Russian Federation (RF) with different climate, geographic, economic and demographic characteristics, to elaborate the risk model for Russian population, and to investigate the traditional and new cardiovascular risk factors. We hypothesize that short and long sleep can be one of the new possible risk factors for cardiovascular diseases (CVD).

The aim of this cross-sectional analysis was to investigate the relationship between self-reported sleep duration and various cardiovascular and metabolic disorders.

\section{Material and methods}

A representative population-based sample of male and female individuals aged 25 to 64 years old was enrolled from 13 Russian regions and cities (North Ossetia - Alania Republic (North Caucasus), Volgograd (South), Vologda (North-West), Voronezh (Centre), Ivanovo (Centre), Kemerovo (West Siberia), Krasnoyarsk (East Siberia), Orenburg (Volga region), Vladivostok (Far East), St Petersburg (North West), Tomsk (West Siberia), Ekaterinburg (West Siberia), Tyumen (Ural)). The survey was accomplished within the period from November 1, 2012 to November 25, 2014. The study was approved by the Institutional Review Boards of 3 main centers: Almazov National Medical Research Centre (St Petersburg), National Research Center for Preventive Medicine (Moscow), Russian Cardiology Research and Production Complex (Moscow) and the associate centers. Detailed study design and enrollment process were described previously elsewhere [17]. All participants gave written informed consent. The response rate was on average $80 \%$.

Overall, 22,258 participants underwent the ESSE-RF survey (8541 men, 38.4\%). Out of all included subjects, 1899 (8.5\%) respondents were excluded due to the missing data: lab tests $(\mathrm{n}=887)$, sleep module $(\mathrm{n}=542)$; inconsistent data $(\mathrm{n}=289)$; 112, 16 and 53 did not report their height, weight and waist/hip ratio, respectively. Therefore, in total 20,359 respondents were included in the final analysis [7,746 men, 38\%; mean age - $48(25 ; 64)$ years].

All subjects underwent a structured interview. The questionnaire was designed on a modular type and included questions regarding social and demographic characteristics, life style and habits (includ- 
ing cigarette smoking, alcohol consumption and physical activity), anamnesis, etc. in accordance with the adapted international methods. Alcohol abuse was considered when $\geq 168 \mathrm{~g}$ of ethanol per week was consumed by men and $\geq 84 \mathrm{~g}$ of ethanol per week by women. The level of physical activity was considered low when a subject reported less than 150 minutes of moderate or less than 75 minutes of intense aerobic physical activity per week, which is the recommended minimum level for adults (walking at medium or high speed, or any other equivalent activity) [18].

History was recorded in the module "diseases" by answering the question "Have your doctor ever told that you have/had the following diseases?" followed by the list of 17 pathologies. In the present paper, we analyzed hypertension, coronary artery disease (CAD), myocardial infarction (MI), stroke (cerebral thrombosis or hemorrhage), obesity, and diabetes mellitus (DM). Participants were asked to provide information about average sleep duration in hours (during one month before the survey): "What was your daily sleep duration? Please, include the naps". Sleep duration of 6 hours or less was considered short [19], $\geq 9$ hours was considered long [20]. Physical examination included anthropometric measurements (waist circumference, height, and weight). Body mass index (BMI) was calculated by Quetelet index — body weight $[\mathrm{kg}] /$ height $^{2}\left[\mathrm{~m}^{2}\right]$. Obesity was diagnosed in case of BMI $\geq 30 \mathrm{~kg} / \mathrm{m}^{2}$. Hypertension was considered when office systolic blood pressure (SBP) was $\geq 140 \mathrm{~mm} \mathrm{Hg}$ and/or diastolic blood pressure (DBP) was $\geq 90 \mathrm{~mm} \mathrm{Hg}$ or antihypertensive therapy was ongoing at the time of the survey. Blood samples were collected according to standardized protocols after $\geq 12$-hour fasting on the day of the survey before the interview and anthropometric measurements were taken. Blood samples were centrifuged at low speed $900 \mathrm{~g}$ for 20 minutes at $+4^{\circ} \mathrm{C}$. Serum was immediately frozen and stored at temperature below $-20^{\circ} \mathrm{C}$ until they were transferred by special logistic services to the laboratory at the responsible participating center.

The following parameters were evaluated: total cholesterol, low density lipoprotein (LDL), high density lipoprotein (HDL), triglycerides, glucose, creatinine, uric acid (Abbot Architect c8000, USA). Glomerular filtration rate (GFR) was estimated by the formula CKD-EPI [21].

\section{Statistical analysis}

All statistical analyses were performed using IBM SPSS Statistics v.21 (USA). Each parameter was tested for normal distribution using the Kolmogo-
rov-Smirnov test. In case of normal distribution the parameters are presented as mean and standard deviation. In case of non-normal distribution the parameters are presented as median and range (minimum; maximum). Comparative analysis of the quantitative variables was performed using Student's t-test. Fisher's exact test and $\chi^{2}$ were used for categorical variables. ANOVA was applied for multiple comparisons. Logistic regression analysis was used to determine predictors among categorical parameters. Sleep duration was included as dependent variable. Cardiovascular diseases (hypertension, CAD etc.), metabolic disorders and other traditional risk factors (age, BMI, office SBP and DBP, smoking, alcohol overuse, cholesterol levels, fasting glucose etc.) were included as independent variables in the univariate analysis. Those factors that had significant predictive value $(p<0.05)$ in the univariate analysis were included in a multivariate model (multinomial logistic regression). Closely correlated factors were not included in the same analysis (e.g. BMI in obesity analysis, glucose level in DM analysis etc.). We estimated odds ratios (ORs) and 95\% confidence intervals (95\% CI) for sleep parameters associated with each risk factor and disorder. Direct standardization of data reported from each region was performed according to the European standards. Differences were considered significant at p-level $<0.05$.

\section{Results}

Mean sleep duration was $7(1 ; 16)$ hours. The life style characteristics were as following: 4440 (21.8\%) smokers, 674 (3.3\%) subjects reported alcohol abuse, low physical activity (less than 5 times a week) -14209 (69.8\%) respondents. About one third of subjects $(\mathrm{n}=7175,35 \%)$ were overweight $\left(25 \leq\right.$ BMI $\left.<30 \mathrm{~kg} / \mathrm{m}^{2}\right)$, every fifth respondent $(\mathrm{n}=4287,21 \%)$ had obesity I degree, the rate of obesity II and III degree was significantly lower ( $\mathrm{n}=1609$, $8 \%$ and $\mathrm{n}=722,4 \%$, respectively). Every $20^{\text {th }}$ patient $(\mathrm{n}=939,5 \%)$ had weight deficit $\left(\mathrm{BMI}<19.9 \mathrm{~kg} / \mathrm{m}^{2}\right)$. Normal fasting glucose level was found in 14961 (74\%) participants, while 2323 (11\%) participants had impaired fasting glucose. Diabetes mellitus was newly diagnosed (based on fasting plasma glucose) in $1960(10 \%)$ patients, while $968(5 \%)$ participants reported previously diagnosed DM. All the subjects were divided into groups based on the sleep duration for the last month: $\leq 6$ hours, $6<$ sleep $<7$ hours, $7-8$ hours, $8<$ sleep $\leq 9$ hours and $>9$ hours of sleep (Tab. I). The majority of subjects reported 7-8 hours $(33.3 \%)$ and from 6 to 7 hours $(32.6 \%)$ of sleep 
Table I. Clinical and demographic characteristics of the study participants depending on sleep duration $(n=20359)$

\begin{tabular}{|c|c|c|c|c|c|c|c|}
\hline Variable & All & $\leq 6$ hours & $\begin{array}{l}6<\text { sleep } \\
<7 \text { hours }\end{array}$ & $7-8$ hours & $\begin{array}{l}8<\text { sleep } \\
\leq 9 \text { hours }\end{array}$ & $>9$ hours & p \\
\hline Age (years) & $\begin{array}{c}48 \\
(25 ; 64)\end{array}$ & $\begin{array}{c}50 \\
(25 ; 64)\end{array}$ & $\begin{array}{c}48 \\
(25 ; 64)\end{array}$ & $\begin{array}{c}48 \\
(25 ; 64)\end{array}$ & $\begin{array}{c}47 \\
(25 ; 64)\end{array}$ & $\begin{array}{c}46 \\
(25 ; 64)\end{array}$ & $<0.001$ \\
\hline $\begin{array}{l}25-34 \\
n=4304(\%)\end{array}$ & 21.1 & 20.8 & 33.6 & 33.08 & 6.78 & 5.62 & \multirow{4}{*}{$\begin{array}{c}\chi^{2}=84.822 \\
p<0.001\end{array}$} \\
\hline $\begin{array}{l}35-44 \\
n=4078(\%)\end{array}$ & 20 & 20.57 & 34.69 & 34.69 & 6.2 & 4.36 & \\
\hline $\begin{array}{l}45-54 \\
n=5681(\%)\end{array}$ & 27.9 & 24.55 & 32.7 & 33.25 & 5.42 & 4.01 & \\
\hline $\begin{array}{l}55-64 \\
n=6296(\%)\end{array}$ & 30.9 & 25.84 & 30.5 & 30.54 & 6.25 & 4.3 & \\
\hline $\begin{array}{l}\text { Gender, men/women } \\
\mathrm{n}(\%)\end{array}$ & $\begin{array}{c}7746(38 \%) \\
12613(62 \%)\end{array}$ & $\begin{array}{l}1714(22.1 \%) \\
3045(24.1 \%)\end{array}$ & $\begin{array}{l}2590(33.4 \%) \\
4057(32.1 \%)\end{array}$ & $\begin{array}{l}2695(34.8 \%) \\
4092(32.4 \%)\end{array}$ & $\begin{array}{l}424(5.4 \%) \\
823(6.5 \%)\end{array}$ & $\begin{array}{l}323(4.1 \%) \\
596(4.7 \%)\end{array}$ & $\begin{array}{c}\chi^{2}=30.6 \\
<0.001\end{array}$ \\
\hline BMI $\left[\mathrm{kg} / \mathrm{m}^{2}\right]$ & $\begin{array}{c}27.4 \\
(15 ; 68)\end{array}$ & $\begin{array}{c}27.8 \\
(15 ; 68)\end{array}$ & $\begin{array}{c}27.0 \\
(15 ; 59)\end{array}$ & $\begin{array}{c}27.4 \\
(15 ; 54)\end{array}$ & $\begin{array}{c}27.0 \\
(15 ; 53)\end{array}$ & $27.0(16 ; 51)$ & $<0.001$ \\
\hline $\begin{array}{l}\text { Waist circumference } \\
\text { [cm] } \\
\text { Men, Women }\end{array}$ & $\begin{array}{l}92(41 ; 160) \\
85(44 ; 160)\end{array}$ & $\begin{array}{c}92(55 ; 150) \\
87.5(44 ; 160)\end{array}$ & $\begin{array}{l}92(52 ; 152) \\
85(50 ; 160)\end{array}$ & $\begin{array}{l}92(41 ; 160) \\
86(45 ; 143)\end{array}$ & $\begin{array}{l}92(48 ; 135) \\
84(57 ; 35.5)\end{array}$ & $\begin{array}{l}93(60 ; 134) \\
85(56 ; 146)\end{array}$ & $\begin{array}{l}<0.05 \\
<0.001\end{array}$ \\
\hline SBP [mm Hg] & $\begin{array}{c}131 \\
(83 ; 233)\end{array}$ & $\begin{array}{c}131 \\
(87 ; 240)\end{array}$ & $\begin{array}{c}130 \\
(80 ; 240)\end{array}$ & $\begin{array}{c}130 \\
(79 ; 240)\end{array}$ & $\begin{array}{c}127 \\
(85 ; 222)\end{array}$ & $\begin{array}{c}128 \\
(83 ; 240) \\
\end{array}$ & $<0.001$ \\
\hline $\mathrm{DBP}[\mathrm{mm} \mathrm{Hg}]$ & $\begin{array}{c}81 \\
(48 ; 137)\end{array}$ & $\begin{array}{c}81 \\
(47 ; 150)\end{array}$ & $\begin{array}{c}81 \\
(40 ; 146)\end{array}$ & $\begin{array}{c}81 \\
(40 ; 150)\end{array}$ & $\begin{array}{c}80 \\
(48 ; 134)\end{array}$ & $\begin{array}{c}81 \\
(44 ; 130) \\
\end{array}$ & $<0.001$ \\
\hline HR [beat per min] & $\begin{array}{c}73 \\
(42 ; 153)\end{array}$ & $\begin{array}{c}73 \\
(43 ; 150)\end{array}$ & $\begin{array}{c}73 \\
(41 ; 165)\end{array}$ & $\begin{array}{c}73 \\
(42 ; 135)\end{array}$ & $\begin{array}{c}73 \\
(45 ; 132)\end{array}$ & $\begin{array}{c}73 \\
(43 ; 128)\end{array}$ & 0.042 \\
\hline $\begin{array}{l}\text { Obesity } \\
n=6648(\%)\end{array}$ & 33 & 36.3 & 31.7 & 31.8 & 30.8 & 32.9 & $\begin{array}{l}\chi^{2}=34.5 \\
p<0.001\end{array}$ \\
\hline $\begin{array}{l}\mathrm{DM} \text { (history) } \\
\mathrm{n}=968(\%)\end{array}$ & 4.7 & 5.4 & 4.4 & 4.5 & 5.1 & 5.2 & $\begin{array}{l}\chi^{2}=7.2 \\
p=0.13\end{array}$ \\
\hline $\begin{array}{l}\text { Hypertension } \\
\mathrm{n}=7670(\%)\end{array}$ & 38 & 40.8 & 36.7 & 37.1 & 34 & 37.6 & $\begin{array}{l}\chi^{2}=30 \\
p<0.001\end{array}$ \\
\hline $\begin{array}{l}\mathrm{CAD} \\
\mathrm{n}=2116(\%)\end{array}$ & 10.0 & 13.4 & 8.7 & 9.7 & 10.3 & 11.9 & $\begin{array}{l}\chi^{2}=70.6 \\
p<0.001\end{array}$ \\
\hline $\begin{array}{l}\mathrm{Ml} \\
\mathrm{n}=443(\%)\end{array}$ & 2.2 & 2.6 & 1.7 & 2.2 & 2.6 & 2.8 & $\begin{array}{l}\chi^{2}=15.8 \\
p=0.003\end{array}$ \\
\hline $\begin{array}{l}\text { Stroke } \\
n=422(\%)\end{array}$ & 2.1 & 2.2 & 1.9 & 2.2 & 2.4 & 1.6 & $\begin{aligned} \chi^{2} & =4.2 \\
p & =0.4\end{aligned}$ \\
\hline $\begin{array}{l}\text { Smoking } \\
\mathrm{n}=4440(\%)\end{array}$ & 21.8 & 24.1 & 21.9 & 20.1 & 18.9 & 24.7 & $\begin{array}{c}\chi^{2}=37.4 \\
<0.001\end{array}$ \\
\hline $\begin{array}{l}\text { Alcohol abuse } \\
n=674(\%)\end{array}$ & 3.3 & 3.2 & 3.2 & 3.2 & 4.1 & 4.2 & 0.2 \\
\hline $\begin{array}{l}\text { Low physical activity } \\
\mathrm{n}=14209(\%)\end{array}$ & 69.8 & 57.3 & 71.6 & 70.0 & 77.4 & 59.4 & $\begin{array}{c}\chi^{2}=24.3 \\
<0.001\end{array}$ \\
\hline
\end{tabular}

$\mathrm{BMI}$ - body mass index; $\mathrm{CAD}$ — coronary artery disease; $\mathrm{DBP}$ — diastolic blood pressure; $\mathrm{DM}$ — diabetes mellitus; $\mathrm{MI}$ - myocardial infarction; $\mathrm{SBP}$ — systolic blood pressure

daily. Almost every $4^{\text {th }}$ participant reported usual short sleep (23.3\%), while only $4.5 \%$ were longsleepers. The similar distribution was found both in men and women. Subjects with longer sleep duration ( $>9$ hours) were younger compared to those sleeping $\leq 6$ hours. Prevalence of short-sleepers increased in elder subjects.
Those sleeping $>8$ hours had the lowest BMI, while short-sleepers had higher BMI values. SBP also increased in short sleepers. Heart rate, glucose and creatinine levels and GFR were also associated with sleep duration, while no significant relation was found between sleep duration and total cholesterol, LDL or triglycerides (Tab. II). At the same time 
Table II. Laboratory parameters of the study participants depending on sleep duration ( $n=20,359$ )

\begin{tabular}{|c|c|c|c|c|c|c|c|}
\hline Variable & All & $\leq 6$ hours & $\begin{array}{c}6<\text { sleep }<7 \\
\text { hours }\end{array}$ & $7-8$ hours & $\begin{array}{c}8<\text { sleep } \leq 9 \\
\text { hours }\end{array}$ & $>9$ hours & p \\
\hline Cholesterol [mmol/L] & $\begin{array}{c}5.4 \\
(1.7 ; 15.7)\end{array}$ & $\begin{array}{c}5.3 \\
(2.1 ; 14)\end{array}$ & $\begin{array}{c}5.3 \\
(2.2 ; 11)\end{array}$ & $\begin{array}{c}5.3 \\
(2.1 ; 12)\end{array}$ & $\begin{array}{c}5.3 \\
(2.4 ; 10)\end{array}$ & $\begin{array}{c}5.3 \\
(2.2 ; 16)\end{array}$ & 0.64 \\
\hline LDL [mmol/L] & $\begin{array}{c}3.3 \\
(0.5 ; 12.1)\end{array}$ & $\begin{array}{c}3.3 \\
(0.5 ; 10)\end{array}$ & $\begin{array}{c}3.3 \\
(0.6 ; 8.6)\end{array}$ & $\begin{array}{c}3.3 \\
(0.7 ; 8.9)\end{array}$ & $\begin{array}{c}3.3 \\
(1.0 ; 7.3)\end{array}$ & $\begin{array}{c}3.2 \\
(0.8 ; 12)\end{array}$ & 0.06 \\
\hline $\mathrm{HDL}[\mathrm{mmol} / \mathrm{L}]$ & $\begin{array}{c}1.4 \\
(0.2 ; 3.8)\end{array}$ & $\begin{array}{c}1.4 \\
(0.4 ; 3.8)\end{array}$ & $\begin{array}{c}1.4 \\
(0.4 ; 3.9)\end{array}$ & $\begin{array}{c}1.4 \\
(0.2 ; 3)\end{array}$ & $\begin{array}{c}1.4 \\
(0.5 ; 2.8)\end{array}$ & $\begin{array}{c}1.3 \\
(0.4 ; 3)\end{array}$ & 0.015 \\
\hline Triglyceride $[\mathrm{mmol} / \mathrm{L}]$ & $\begin{array}{c}1.2 \\
(0.1 ; 16.1)\end{array}$ & $\begin{array}{c}1.2 \\
(0.1 ; 14)\end{array}$ & $\begin{array}{c}1.2 \\
(0.1 ; 16)\end{array}$ & $\begin{array}{c}1.2 \\
(0.2 ; 16)\end{array}$ & $\begin{array}{c}1.2 \\
(0.3 ; 10)\end{array}$ & $\begin{array}{c}1.2 \\
(0.3 ; 19)\end{array}$ & 0.15 \\
\hline Glucose [mmol/L] & $\begin{array}{c}5.1 \\
(2.5 ; 25.5)\end{array}$ & $\begin{array}{c}5.1 \\
(2.5 ; 24)\end{array}$ & $\begin{array}{c}5.1 \\
(2.5 ; 25.5)\end{array}$ & $\begin{array}{c}5.1 \\
(2.7 ; 22.5)\end{array}$ & $\begin{array}{c}5.0 \\
(3 ; 24)\end{array}$ & $\begin{array}{c}5.1 \\
(2.6 ; 21)\end{array}$ & 0.024 \\
\hline Creatinine $[\mu \mathrm{mol} / \mathrm{L}]$ & $\begin{array}{c}69.3 \\
(31 ; 918)\end{array}$ & $\begin{array}{c}67.0 \\
(33 ; 734)\end{array}$ & $\begin{array}{c}67.4 \\
(31 ; 700)\end{array}$ & $\begin{array}{c}68.0 \\
(32 ; 653)\end{array}$ & $\begin{array}{c}67.0 \\
(39 ; 461)\end{array}$ & $\begin{array}{c}67.0 \\
(41 ; 918)\end{array}$ & 0.022 \\
\hline $\begin{array}{l}\mathrm{GFR} \\
{[\mu \mathrm{mol} / \mathrm{L} / \mathrm{min} \times 1.73]}\end{array}$ & $\begin{array}{c}100 \\
(5.4 ; 173)\end{array}$ & $\begin{array}{c}99.5 \\
(7 ; 152)\end{array}$ & $\begin{array}{c}100.4 \\
(7 ; 173)\end{array}$ & $\begin{array}{c}100.0 \\
(6 ; 166)\end{array}$ & $\begin{array}{c}100.5 \\
(9 ; 138)\end{array}$ & $\begin{array}{c}100.0 \\
(5 ; 138)\end{array}$ & 0.021 \\
\hline Uric Acid, [mmol/L] & $346(81 ; 1000)$ & $340(110 ; 800)$ & $350(100 ; 1000)$ & $341(81 ; 850)$ & $344(163 ; 650)$ & $330(126 ; 810)$ & $<0.05$ \\
\hline Men/Women & $270(80 ; 1130)$ & $270(80 ; 777)$ & $270(80 ; 1030)$ & $267(80 ; 925)$ & $260(110 ; 1130)$ & $260(89 ; 676)$ & $>0.05$ \\
\hline
\end{tabular}

HDL — high density lipoprotein; GFR — glomerular filtration rate; LDL — low density lipoprotein

Table III. Association between sleep duration and cardiovascular diseases (OR; 95\% CI)

\begin{tabular}{|c|c|c|c|c|c|c|c|}
\hline \multicolumn{2}{|c|}{ Sleep duration } & \multirow{2}{*}{$\begin{array}{c}\text { Obesity } \\
1.2^{* * *} \\
(1.1 ; 1.3)\end{array}$} & \multirow{2}{*}{$\begin{array}{c}\begin{array}{c}\text { Diabetes } \\
\text { Mellitus }\end{array} \\
1.2^{*} \\
(1.02 ; 1.4)\end{array}$} & \multirow{2}{*}{$\begin{array}{c}\text { Hypertension } \\
1.2^{* * *} \\
(1.1 ; 1.3)\end{array}$} & \multirow{2}{*}{$\begin{array}{c}\begin{array}{c}\text { Coronary Artery } \\
\text { Disease }\end{array} \\
1.4^{* * *} \\
(1.3 ; 1.6)\end{array}$} & \multirow{2}{*}{$\begin{array}{c}\begin{array}{c}\text { Myocardial } \\
\text { Infarction }\end{array} \\
1.2 \\
(0.9 ; 1.5)\end{array}$} & \multirow{2}{*}{$\begin{array}{c}\text { Stroke } \\
1.0 \\
(0.8 ; 1.3)\end{array}$} \\
\hline \multirow{2}{*}{$\leq 6 \mathrm{~h}$} & Model 1 & & & & & & \\
\hline & Model 2 & $\begin{array}{c}1.1^{* *} \\
(1.04 ; 1.3)\end{array}$ & $\begin{array}{c}1.0 \\
(0.9 ; 1.2)\end{array}$ & $\begin{array}{c}1.1 \\
(1.0 ; 1.2)\end{array}$ & $\begin{array}{c}1.3^{* * *} \\
(1.2 ; 1.5)\end{array}$ & $\begin{array}{c}1.1 \\
(0.9 ; 1.5)\end{array}$ & $\begin{array}{c}0.9 \\
(0.7 ; 1.2)\end{array}$ \\
\hline \multirow{2}{*}{$\begin{array}{l}6<\text { sleep } \\
<7 \mathrm{~h}\end{array}$} & Model 1 & $\begin{array}{c}1.0 \\
(0.9 ; 1.1)\end{array}$ & $\begin{array}{c}1.0 \\
(0.9 ; 1.2)\end{array}$ & $\begin{array}{c}1.0 \\
(0.9 ; 1.1)\end{array}$ & $\begin{array}{c}0.8^{*} \\
(0.7 ; 0.9)\end{array}$ & $\begin{array}{c}0.7^{*} \\
(0.6 ; 0.9)\end{array}$ & $\begin{array}{c}0.9 \\
(0.7 ; 1.1)\end{array}$ \\
\hline & Model 2 & $\begin{array}{c}1.0 \\
(0.9 ; 1.1)\end{array}$ & $\begin{array}{c}1.0 \\
(0.9 ; 1.2)\end{array}$ & $\begin{array}{c}1.0 \\
(0.9 ; 1.1)\end{array}$ & $\begin{array}{c}0.9 \\
(0.8 ; 1.0)\end{array}$ & $\begin{array}{c}0.8 \\
(0.6 ; 1.0)\end{array}$ & $\begin{array}{c}0.9 \\
(0.7 ; 1.1)\end{array}$ \\
\hline \multicolumn{2}{|l|}{$7-8 \mathrm{~h}$} & \multicolumn{6}{|c|}{ Reference variable } \\
\hline \multirow{2}{*}{$\begin{array}{l}8<\text { sleep } \\
\leq 9 \mathrm{~h}\end{array}$} & Model 1 & $\begin{array}{c}1.0 \\
(0.8 ; 1.1)\end{array}$ & $\begin{array}{c}1.1 \\
(0.9 ; 1.5)\end{array}$ & $\begin{array}{c}0.8^{*} \\
(0.7 ; 0.9)\end{array}$ & $\begin{array}{c}1.1 \\
(0.9 ; 1.3)\end{array}$ & $\begin{array}{c}1.2 \\
(0.8 ; 1.7)\end{array}$ & $\begin{array}{c}1.1 \\
(0.7 ; 1.7)\end{array}$ \\
\hline & Model 2 & $\begin{array}{c}1.0 \\
(0.9 ; 1.2)\end{array}$ & $\begin{array}{c}1.2 \\
(0.9 ; 1.6)\end{array}$ & $\begin{array}{c}0.9 \\
(0.8 ; 1.1)\end{array}$ & $\begin{array}{c}1.1 \\
(0.9 ; 1.4)\end{array}$ & $\begin{array}{c}1.3 \\
(0.9 ; 2.0)\end{array}$ & $\begin{array}{c}1.2 \\
(0.8 ; 1.8)\end{array}$ \\
\hline \multirow{2}{*}{$>9 \mathrm{~h}$} & Model 1 & $\begin{array}{c}1.0 \\
(0.9 ; 1.2)\end{array}$ & $\begin{array}{c}1.2 \\
(0.9 ; 1.6)\end{array}$ & $\begin{array}{c}1.0 \\
(0.9 ; 1.2)\end{array}$ & $\begin{array}{c}1.3^{*} \\
(1.02 ; 1.6)\end{array}$ & $\begin{array}{c}1.3 \\
(0.8 ; 2.0)\end{array}$ & $\begin{array}{c}0.8 \\
(0.4 ; 1.3)\end{array}$ \\
\hline & Model 2 & $\begin{array}{c}1.1 \\
(0.9 ; 1,3)\end{array}$ & $\begin{array}{c}1.3 \\
(0.9 ; 1.8)\end{array}$ & $\begin{array}{c}1.1 \\
(1.0 ; 1.3)\end{array}$ & $\begin{array}{c}1.5^{* *} \\
(1.1 ; 1.8)\end{array}$ & $\begin{array}{c}1.6^{*} \\
(1.02 ; 2.4)\end{array}$ & $\begin{array}{c}0.8 \\
(0.5 ; 1.4)\end{array}$ \\
\hline
\end{tabular}

Model 1 - unadjusted; Model 2 - adjusted for sex, age, body mass index, office blood pressure, smoking and low physical activity; ${ }^{*} \mathrm{p}<0.05 ;{ }^{* *} \mathrm{p}<0.01$; ${ }^{* * *} \mathrm{p}<0.001$

HDL cholesterol was significantly lower in longsleepers. Gender-specific differences were shown for uric acid: the highest levels of uric acid were found in men sleeping 6-7 hours.

Short- and long-sleepers less often had low physical activity $\left(\chi^{2}=24.3 ; \mathrm{p}<0.001\right)$ and smoked more often $\left(\chi^{2}=37.4 ; \mathrm{p}<0.001\right)$ than normal sleepers. The rate of alcohol abuse was not significant in groups with different sleep duration.
We also found (Tab. I) a U-shaped relation between sleep duration and other cardiovascular risk factors and diseases: short- and long-sleepers had higher incidence of obesity, hypertension, CAD and MI. There were no significant differences for DM and stroke.

Logistic regression showed a higher probability of obesity, hypertension and CAD (Tab. III) in shortsleepers. Six-to-seven-hour sleep was associated with 
lower probability of CAD and MI. Moreover, sleep duration from 8 to 9 hours was associated with the lowest risk of hypertension $(\mathrm{OR}=0.8$; CI: $0.7-0.9$, $\mathrm{p}=0.037)$. At the same time sleep over 9 hours was associated with the higher risk of $\mathrm{CAD}(\mathrm{OR}=1.3$; CI: $1.02-1.6, p=0.043)$. After adjustment for sex, age, BMI, office blood pressure, smoking and low physical activity the associations were weaker but remained significant (Tab. II). Compared with subjects sleeping $7-8 \mathrm{~h}$, the multivariable OR $(95 \% \mathrm{CI})$ was higher for obesity in short-sleepers - 1.1 (1.03; 1.2), meanwhile the association was U-shaped for $\mathrm{CAD}$, and J-shaped for MI. No associations were found for hypertension, DM and stroke.

\section{Discussion}

Based on the data from the epidemiological study ESSE-RF the median sleep duration in Russian citizens is 7 hours. Almost every 4th citizen $(23.3 \%)$ is a short-sleeper. This conforms with the results of the National Health Interview Survey [22] - 29\% (8\% subjects sleeping $\leq 5 \mathrm{~h} /$ day and $21 \%$ those sleeping $6 \mathrm{~h} /$ day), but the prevalence of short-sleepers among Russian population is significantly higher than the data reported in the Sleep Heart Health Study (9.2\%) [23]. These differences are due to the various approaches for sleep assessment. The first study, similarly to ours, estimated average daily sleep duration, while the second one identified mean sleep duration on weekends and workdays. In both studies, the proportion of long-sleepers is greater than in our cohort - $4.5 \%$ vs. $7.6 \%$ and $9 \%$ in SHHS and NHIS, respectively.

Based on our analysis, short sleep duration is associated with the increased risk of obesity, hypertension and $\mathrm{CAD}$. The incidence of $\mathrm{CAD}$ and $\mathrm{MI}$ was higher for both short- and long-sleepers. No significant differences between subjects with various sleep duration were found regarding alcohol abuse. However, short- and long-sleepers reported lower prevalence of low physical activity and smoked more frequently. Therefore, our data do not confirm any definite and unidirectional relationship between sleep duration and cardiovascular risk factors.

The data on sleep duration and sleep quality in Russian population is lacking. The ESSE-RF is the first study which estimated sleep duration in Russia. At the same time, sleep quality was assessed in few other studies. Participants of the HAPIEE study 45-69 years old reported frequent spontaneous sleep interruptions in 20\%; and unsatisfying sleep in 10\% respondents [24]. Sleep quality was associated with angina, arthritis and depression in the analysis of the data from Russian population performed by the SAGE investigators [25].

Unlike other studies, our analysis did not show associations between short or long sleep duration with DM, hypertension or stroke. The Sleep Heart Health Study demonstrated higher risk for hypertension [23] for both short- $(\mathrm{OR}=1.66$, CI 1.35-2.04, $\mathrm{p}<0.0001)$ and long-sleepers $(\mathrm{OR}=1.30$, CI: $1.04-1.62, p<0.0001)$. In addition, both shortsleepers $(\mathrm{OR}=2.01, \mathrm{CI}: 1.5-2.70, \mathrm{p}<0.005)$ and long-sleepers $(\mathrm{OR}=2.22, \mathrm{CI}: 1.69-2.91, \mathrm{p}<0.005)$ had higher risk of stroke [19]. The meta-analyses of 24 prospective cohorts including 474684 participants with short $(\leq 5-6 \mathrm{~h})(\mathrm{OR}=1.15$, CI: $1.00-1.31$, $\mathrm{p}=0.047)$ and long sleep duration $(>8-9 \mathrm{~h})$ $(\mathrm{OR}=1.65, \mathrm{CI}: 1.45-1.87, \mathrm{p}<0.0001)$ showed higher risk of fatal and non-fatal stroke compared to reference subjects sleeping $7-8 \mathrm{~h}$ per day [7].

\section{Limitations of the study}

One of the most significant limitations of our study is the absence of objective (instrumental) verification of sleep duration, although according to multiple studies self-reports correlate with the actigraphy data [26]. Another limiting factor is the cross-sectional design of our study. Short or long sleep duration is not always habitual. It might result from common sleep disorders (e.g., decrease in sleep time and low sleep quality in insomniacs, sleep apnea-related significant reduction in sleep quality). We also did not analyze the factors limiting sleep duration - the duration of work time, the time which subjects waste to get to the workplace, work schedule, chronotype and etc., because these factors were not included in the structured interview according to the primary design of the ESSE-RF study.

\section{Conclusions}

Our data support the concept of the relation between sleep and cardiometabolic disorders and provide additional evidence on the unfavorable impact of extreme sleep duration variations. We demonstrated that sleep duration is highly variable in Russian population with significant prevalence of short sleepers. However, unlike published data, in our cohort not all cardiovascular diseases were associated with sleep duration. The multivariable odds ratio was higher for obesity in short-sleepers compared with those who slept 7-8 hours. Meanwhile the U-shaped association was found for coronary artery disease. Association with myocardial infarction is characterized 
by the J-shaped relation. There were no associations for hypertension, diabetes mellitus and stroke. We believe that our results provide important information for the understanding of sleep epidemiology and relation between sleep and cardiovascular risk.

\section{Acknowledgements}

The publication was supported by the Grant of the President of Russian Federation for the Leading Scientific Schools of Russia HW-5508.2018.7 (agreement No. 075-15-2019-161, 23.05.2019).

On behalf of the ESSE-RF study investigators from Russia: St Petersburg, Almazov National Medical Research Centre: Elena Baranova; Moscow, Russian Cardiology Research and Production Complex: Elena Oshchepkova; National Research Center for Preventive Medicine: Raphael Oganov, Galina Muromtseva, YuliaBalanova; Alania, North Ossetian State Medical Academy: George Tolparov; Vladivostok, Tihookeanskij State Medical Academy: Vera Nevzorova, Natalia Kulakova; Volgograd, Volgograd State Medical University: Sergey Nedogoda; Vologda, Institute of Territory Socio-economic Development: Alexandra Shabunova; Voronezh, Voronezh State Medical University named after N.N. Burdenko: Galina Furmenko; Ivanovo, Ivanovo cardiologic dispensary: Olga Belova; Krasnoyarsk, Krasnoyarsk State Medical University: Yuri Greenstein, Marina Petrova; Orenburg, Orenburg State Medical University: Roman Libis; Samara, Samara regional clinical cardiology dispensary: Dmitry Dupliakov; Tomsk, Tomsk Research Institute of Cardiology: Vladimir Kaveshnikov; Tyumen, Tyumen State Medical Academy: Alexey Efanov; Kemerovo, Research Institute for Complex Problems of Cardiovascular Diseases: Galina Artamonova, Elena Indukaeva.

\section{Conflict of interest}

None declared.

\section{References}

1. Bochkarev MV, Korostovtseva LS, Sviryaev YV. [Sleep quality and duration cardiovascular diseases: is there an association?]. Arterial'naya Gypertenziya. 2014; 19(450): 461.

2. Williams JA, Zimmerman FJ, Bell JF. Norms and trends of sleep time among US children and adolescents. JAMA Pediatr. 2013; 167(1): 55-60, doi: 10.1001/jamapediatrics.2013.423, indexed in Pubmed: 23403646.

3. Hirshkowitz M, Whiton K, Albert SM, et al. National Sleep Foundation's sleep time duration recommendations: methodology and results summary. Sleep Health. 2015; 1(1): 40-43, doi: 10.1016/j. sleh.2014.12.010, indexed in Pubmed: 29073412.

4. Mukherjee S, Patel SR, Kales SN, et al. American Thoracic Society ad hoc Committee on Healthy Sleep. An Official American Thoracic Society Statement: The Importance of Healthy Sleep. Recommendations and Future Priorities. Am J Respir Crit Care Med. 2015;
191(12): 1450-1458, doi: 10.1164/rccm.201504-0767ST, indexed in Pubmed: 26075423.

5. Watson NF, Badr MS, Belenky G, et al. Consensus Conference Panel. Joint Consensus Statement of the American Academy of Sleep Medicine and Sleep Research Society on the Recommended Amount of Sleep for a Healthy Adult: Methodology and Discussion. J Clin Sleep Med. 2015; 11(8): 931-952, doi: 10.5664/jcsm.4950, indexed in Pubmed: 26235159.

6. Kripke DF, Garfinkel L, Wingard DL, et al. Mortality associated with sleep duration and insomnia. Arch Gen Psychiatry. 2002; 59(2): 131-136, doi: 10.1001/archpsyc.59.2.131, indexed in Pubmed: 11825133.

7. Cappuccio FP, Cooper D, D'Elia L, et al. Sleep duration predicts cardiovascular outcomes: a systematic review and meta-analysis of prospective studies. Eur Heart J. 2011; 32(12): 1484-1492, doi: 10.1093/eurheartj/ehr007, indexed in Pubmed: 21300732.

8. Stamatakis KA, Punjabi NM. Effects of sleep fragmentation on glucose metabolism in normal subjects. Chest. 2010; 137(1): 95-101, doi: 10.1378/chest.09-0791, indexed in Pubmed: 19542260.

9. St-Onge MP. The role of sleep duration in the regulation of energy balance: effects on energy intakes and expenditure. J Clin Sleep Med. 2013; 9(1): 73-80, doi: 10.5664/jcsm.2348, indexed in Pubmed: 23319909.

10. Reutrakul S, Van Cauter E. Sleep influences on obesity, insulin resistance, and risk of type 2 diabetes. Metabolism. 2018; 84: 56-66, doi: 10.1016/j.metabol.2018.02.010, indexed in Pubmed: 29510179.

11. Almeida FR, Perry JC, Futuro-Neto HA, et al. Cardiovascular function alterations induced by acute paradoxical sleep deprivation in rats. Clin Exp Hypertens. 2014; 36(8): 567-571, doi: 10.3109/106419 63.2014.881843, indexed in Pubmed: 24678694.

12. Haack M, Serrador J, Cohen D, et al. Increasing sleep duration to lower beat-to-beat blood pressure: a pilot study. J Sleep Res. 2013; 22(3): 295-304, doi: 10.1111/jsr.12011, indexed in Pubmed: 23171375.

13. Petrov ME, Kim Y, Lauderdale D, et al. Longitudinal associations between objective sleep and lipids: the CARDIA study. Sleep. 2013; 36(11): 1587-1595, doi: 10.5665/sleep.3104, indexed in Pubmed: 24179290.

14. Hu L, Zhang B, Zhou W, et al. Sleep duration on workdays or nonworkdays and cardiac-cerebral vascular diseases in Southern China. Sleep Med. 2018; 47: 36-43, doi: 10.1016/j.sleep.2017.11.1147, indexed in Pubmed: 29880146.

15. Kiejna A, Rymaszewska J, Wojtyniak B, et al. Characteristics of sleep disturbances in Poland - results of the National Health Interview Survey. Acta Neuropsychiatr. 2004; 16(3): 124-129, doi: 10.1111/j.0924-2708.2004.00063.x, indexed in Pubmed: 26984163.

16. Cappuccio FP, Stranges S, Kandala NB, et al. Gender-specific associations of short sleep duration with prevalent and incident hypertension: the Whitehall II Study. Hypertension. 2007; 50(4): 693-700, doi: 10.1161/HYPERTENSIONAHA.107.095471, indexed in Pubmed: 17785629.

17. Rotar O, Boyarinova M, Orlov A, et al. Metabolically healthy obese and metabolically unhealthy non-obese phenotypes in a Russian population. Eur J Epidemiol. 2017; 32(3): 251-254, doi: $10.1007 /$ s10654-016-0221-z, indexed in Pubmed: 28039558.

18. Piepoli MF, Hoes AW, Agewall S, et al. ESC Scientific Document Group. 2016 European Guidelines on cardiovascular disease prevention in clinical practice: The Sixth Joint Task Force of the European Society of Cardiology and Other Societies on Cardiovascular Disease Prevention in Clinical Practice (constituted by representatives of 10 societies and by invited experts)Developed with the special contribution of the European Association for Cardiovascular Prevention \& Rehabilitation (EACPR). Eur Heart J. 2016; 37(29): 2315-2381, doi: 10.1093/eurheartj/ehw106, indexed in Pubmed: 27222591.

19. Grandner MA, Patel NP, Gehrman PR, et al. Problems associated with short sleep: bridging the gap between laboratory and epidemiological studies. Sleep Med Rev. 2010; 14(4): 
239-247, doi: 10.1016/j.smrv.2009.08.001, indexed in Pubmed: 19896872.

20. Grandner MA, Drummond SPA. Who are the long sleepers? Towards an understanding of the mortality relationship. Sleep Med Rev. 2007; 11(5): 341-360, doi: 10.1016/j.smrv.2007.03.010, indexed in Pubmed: 17625932.

21. Levey AS, Stevens LA, Schmid CH, et al. CKD-EPI (Chronic Kidney Disease Epidemiology Collaboration). A new equation to estimate glomerular filtration rate. Ann Intern Med. 2009; 150(9): 604-612, doi: 10.7326/0003-4819-150-9-200905050-00006, indexed in Pubmed: 19414839.

22. Sabanayagam C, Shankar A. Sleep duration and cardiovascular disease: results from the National Health Interview Survey. Sleep. 2010; 33(8): 1037-1042, doi: 10.1093/sleep/33.8.1037, indexed in Pubmed: 20815184.
23. Gottlieb DJ, Redline S, Nieto FJ, et al. Association of usual sleep duration with hypertension: the Sleep Heart Health Study. Sleep. 2006; 29(8): 1009-1014, doi: 10.1093/sleep/29.8.1009, indexed in Pubmed: 16944668.

24. Gafarov VV, Gagulin IV, et al. GromovaEA, Sleep disorders in 45-69-year-old population in Russia/Siberia (Epidemiology study). Int J Med Med Sci. 2013; 3(6): 470-475.

25. Koyanagi Ai, Garin N, Olaya B, et al. Chronic conditions and sleep problems among adults aged 50 years or over in nine countries: a multi-country study. PLoS One. 2014; 9(12): e114742, doi: 10.1371/ journal.pone.0114742, indexed in Pubmed: 25478876.

26. Lauderdale DS, Knutson KL, Yan LL, et al. Self-reported and measured sleep duration: how similar are they? Epidemiology. 2008; 19(6): 838-845, doi: 10.1097/EDE.0b013e318187a7b0, indexed in Pubmed: 18854708. 